\title{
Construction of a Transformer DGA Health Index Based on DGA Screening Processes
}

\author{
Michael Hosseini \\ Institute for Energy and Environment \\ Department of Electronic and Electrical \\ Engineering \\ University of Strathclyde \\ Glasgow, UK \\ Michael.Hosseini@strath.ac.uk
}

\author{
Brian G. Stewart \\ Institute for Energy and Environment \\ Department of Electronic and Electrical \\ Engineering \\ University of Strathclyde \\ Glasgow, UK
}

Nick Torenvliet

Specialized Tooling

Bruce Power

Ontario, Canada

\author{
Martin Kearns \\ Central Technical Organisation \\ Nuclear Generation \\ EDF Energy \\ Glasgow, UK \\ Martin.kearns@edf-energy.com
}

\begin{abstract}
An automated transformer dissolved gas analysis evaluation system based on the recently released IEEE Standard C57.104-2019 for mineral oil-immersed transformers is implemented and its outputs demonstrated. The system includes diagnostic capabilities using the Duval Triangles 1-4-5 and Rogers Ratio method. In addition, a Health Index derived from the above Standards is proposed as a compact, yet informative metric for tracking transformer health. Finally, some of the challenges in developing such an implementation are highlighted.
\end{abstract}

Keywords-transformers, condition monitoring, dissolved gas analysis, DGA, C57.104

\section{INTRODUCTION}

During transformer operations, different gases can be generated depending on various underlying mechanisms and on the materials present within the transformer. The measurement and analysis of the gas levels collected from within the transformer can provide insight into which of these mechanisms may be present, as well as their indicative scale and severity e.g. [1]-[3]. This is the topic of transformer Dissolved Gas Analysis (DGA). Measurement of DGA provides a relatively unobtrusive method to detect potential maloperation within the transformer via either offsite laboratory testing of oil samples, or in situ measuring devices.

The IEEE recently published the C57.104-2019 Guide for the interpretation of gases generated in mineral oil-immersed transformers which focusses on laboratory-based DGA testing [1]. The proposed method inspects up to seven key gases: hydrogen $(\mathrm{H} 2)$, methane $(\mathrm{CH} 4)$, ethane $(\mathrm{C} 2 \mathrm{H} 6)$, ethylene $(\mathrm{C} 2 \mathrm{H} 4)$, acetylene $(\mathrm{C} 2 \mathrm{H} 2)$, carbon monoxide $(\mathrm{CO})$, and carbon dioxide $\mathrm{CO} 2$ ). For each gas, four thresholds are checked against from four respective tables. Table 1 and Table 2 within [1] compare the absolute gas levels against their thresholds, with Table 2 being the higher threshold. Table 3 in [1] compares the change in gas levels between two subsequent samples. Table 4 in [1] is more complicated and is described as 'multi-points rate analysis'. It is a measure of the projected annual change in gas levels based on a linear regression from a subset of the gas samples.

Depending on the results of these checks, for each gas, a Status Level is assigned. The definitions of the Status Levels are quoted directly and are as defined below [1]:
- DGA Status 1: Screening DGA results are acceptable. Continue routine operation.

- DGA Status 2: Incipient or modest recent gas production or moderately elevated gas level. Resample for confirmation and monitor possible gas evolution.

- DGA Status 3: High gas levels or continuing significant gas production. Mitigative actions or other responses should be considered (i.e., continuous monitoring).

Where different gases output different Status Levels, the worst case is selected to represent the transformer. The method to select the appropriate Status Level is shown in Fig. 1 [1] which is derived from the procedure described in the Guide. Where a sample indicates Status Level 2 or 3, further diagnostic analysis is recommended to investigate the potential cause.

It should be noted that the Guide emphasises that this DGA Status Level is not equivalent to the Transformer Condition given some of the shortcomings inherent to DGA as well as the method of establishing the chosen thresholds for each of the Tables. Nevertheless, the work presented in this paper seeks to create an informative DGA-based Health Index (HI) based on an automated implementation of the Guide as a complimentary decision-support tool to experienced engineers in the asset-management of transformers.

The subsequent section will detail the automation process, including the derivation into a Health Index. This is followed by a short case study demonstrating the motivation for conversion of the Guide's original output into a Health Index, as well as a discussion of some of the challenges in implementing the method outlined in [1].

\section{AUTOMATED IMPLEMENTATION}

\section{A. Derivation of Status Level}

The IEEE Guide outlines the process of deriving a Status Level multiple times [1]. However, they are not all consistent. The stepped procedure provided in the Guide is used in this implementation. The flow chart in the Guide was not used, as though it is the simplest, it lacked information regarding the "resampling" protocol. According to the stepped procedure, 
this resampling protocol occurs when absolute gas levels are low, yet either the relative change in gas levels, or the projected change in gas levels exceed their respective thresholds in Tables 3 and 4. In this case, a confirmation sample taken within one month is recommended. This resample temporarily replaces the original sample, and the relevant metrics are recalculated. If the result is a Status Level 1 , then the original sample is deemed anomalous and disregarded henceforth. However, if the resample confirms an elevated Status Level, then both samples are retained. The interpreted procedure is shown in Fig. 1. Where the description in the Guide was not explicit, reasonable assumptions have been made, and highlighted in grey within the figure.

Table 1 and Table 2 in [1], which compare against the absolute gas levels of a given sample, are trivial to implement. Table 3 requires the relative change in gas levels between two gas samples. This has the complication that during the resampling procedure, the application of Table 3 requires the relative change in gas levels between the current sample and the third most recent sample (i.e. not the usual second recent sample). An extension of this is when a sample is considered anomalous, in which case it should not be used as a point of comparison. This implementation simply uses a flag to indicate whether a sample is a 'valid' point of comparison. Then, each sample is iteratively compared with the most recent previous sample that has the flag indicating it is valid.

The process for calculating Table 4 values is more convoluted and is shown in Fig. 2. The criteria in [1] for a valid selection of samples to calculate the annualised projected change in gas levels via the application of a linear regression is that there must be between 3 and 6 samples which span a duration between 4 and 24 months. Samples which have been deemed invalid should not be included. Likewise, the resampling procedure outlined above for Table 3 , where a sample is temporarily ignored until its subsequent sample confirms its output, still applies.

It should be noted that the derivation of Status Level is done for each of the available gases, and that the worst-case output is selected to represent the sample. This output represents a simplified implementation of [1].

However, as the subsequent case study will aim to demonstrate, the metric of the Status Level alone is too coarse to be used as a Health Index. A single metric from a single gas can cause a Status Level 3, which is then indistinguishable from a case where all gases fail across all metrics for example. This paper proposes a simple and intuitive modification to provide a more granular and informative metric.

\section{B. Derivation of Health Index}

The Status Level is based on the worst-case across all the gases. However, this can mask overall trending indicators. For example, no distinction is made between a sample with all gases indicating a Status Level 3, and a sample where only one gas indicates as such. In contrast, using the average Status Level across all gases can underemphasise the importance of even a single gas being highly anomalous. Instead, the mean average of the worst-case and the mean average across all gases is used to balance both concerns.

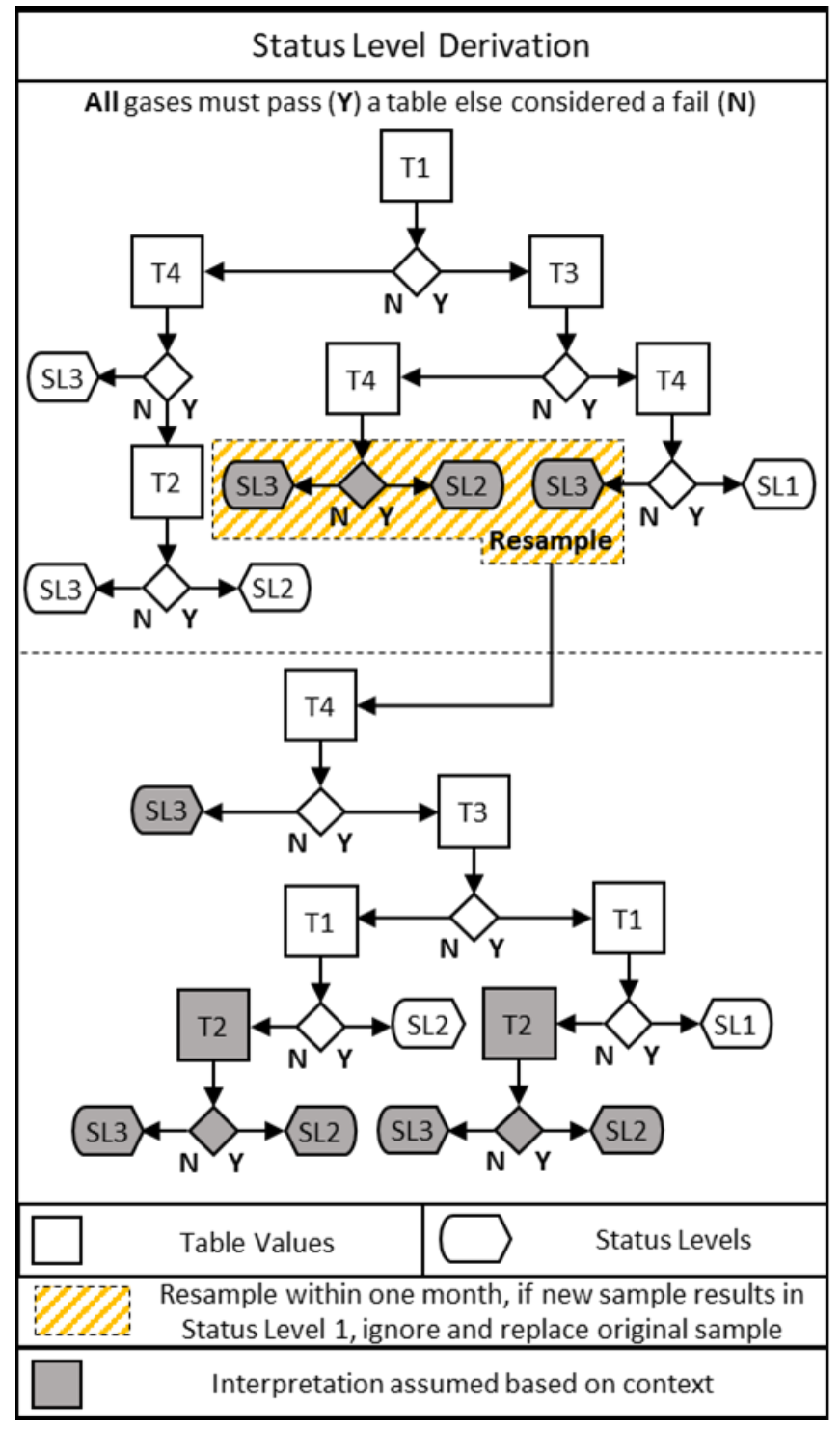

Fig. 1. Interpreted derivation on Status Level based on [1]

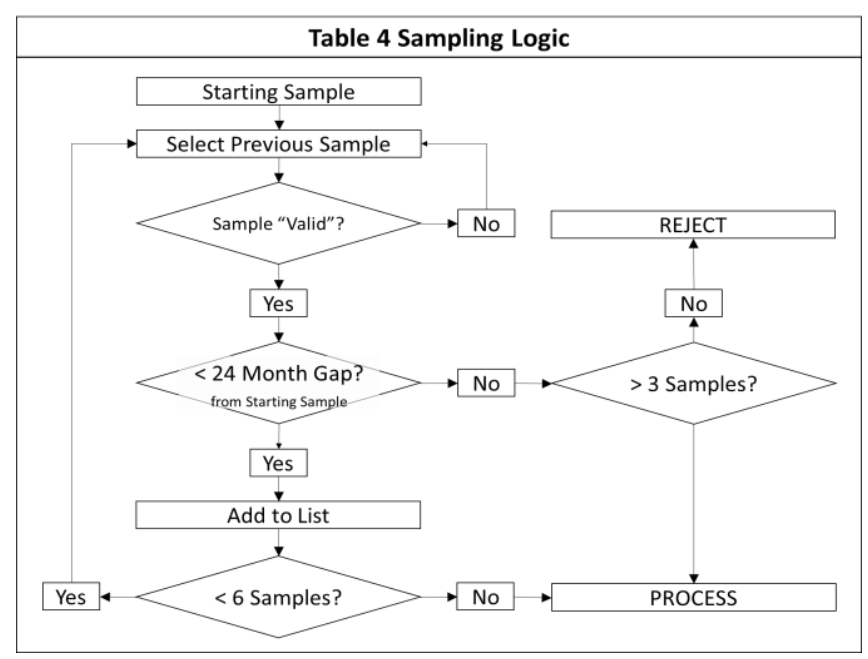

Fig. 2. Implemented automation of [1]'s Table 4 sampling selection

Furthermore, it is proposed that the Status Levels are mapped to a 0:1 Health Index Scale, where 0 represents Status Level 3 , and 1 represents Status Level 1. The intermediate values represent Status Level 2. Exceeding the threshold stated in 
Table 3 is equivalent to a value 0.5 for the Health Index. Table 1 and Table 2 are linearly interpolated to map to the intermediate points along the 0:1 Health Index scale. The rationale is that Table 1 and Table 2 are defined as the $90^{\text {th }} \%$ and $95^{\text {th }} \%$ of the gas concentrations based on the dataset used in [1], respectively. Where both Table 1 and Table 3 are exceeded, the minimum of the two calculated values is used. This is shown in Fig. 3.

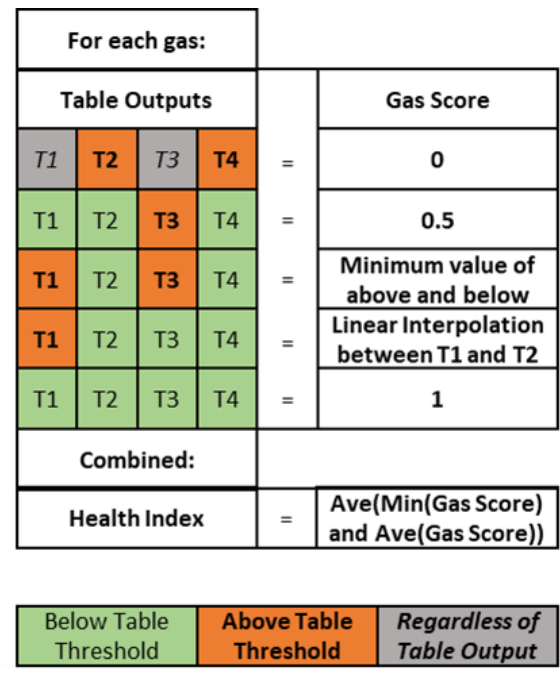

Fig. 3. Proposed derivation of Health Index from Status Levels

\section{Implementation of Diagnostic Analyses}

The IEEE Guide recommends conducting further diagnostic analyses on samples where the Status Level is 2 or 3 . Within the main body of the Guide, Rogers Ratio method [4] and Duval Triangle 1 [5] are described. The Annexes also describe Duval Triangles 4 and 5 [6], Duval Pentagons 1 and 2, the Key Gas method, and Doernenburg Ratios method. From this list, this implementation includes only Duval Triangles 1-4-5 and Rogers Ratio method.

The Duval Triangle method has been widely used in the literature and will not be explained in detail [1], [2], [5], [6]. Each of the Duval Triangles are a ternary plot of three gases on a ratio-scale, i.e. any given sample on the plot will have the three gases sum to $100 \%$. Each Duval Triangle has its own set of three gases, and its own regions corresponding to a particular likely diagnosis. Finally, Duval Triangle 4 and 5 are extensions to Duval Triangle 1 and are applicable depending on the output of Duval Triangle 1. This is shown in Fig. 4. It should be noted that it is emphasised that some of the outputs are not guaranteed. For example, it is stated that the probability of having carbonisation is $80 \%$ even if the sample lies in the region corresponding to said diagnosis.

The automated implementation simply determines the corresponding diagnosis based on the regions outlined in [1]. The outputs are then plotted on figures similar to Fig. 4, as well as a categorical plot against time as shown in Fig. 5.

The Rogers Ratio method is a well-established simple table that uses three pairs of ratios to suggest a fault diagnosis [1], [4]. Even within [1], it is stated that a limitation of its use is that it cannot identify faults in a relatively large number of DGA results. This corroborates with the outputs from the case studies considered by the authors when adopting this methodology. The automated implementation uses a categorical plot against time to display the calculated suggested fault diagnosis as shown in Fig. 5.

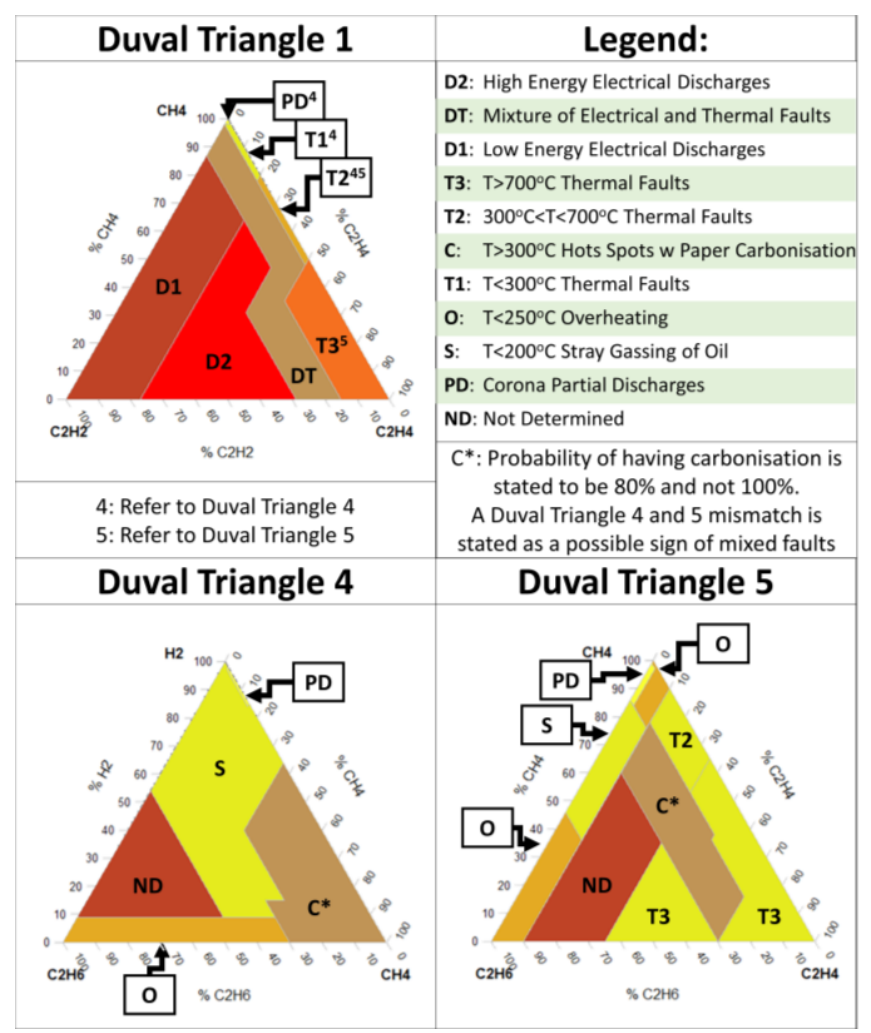

Fig. 4. Duval Triangles 1-4-5, based on [1]

\section{CASE StUdy RESUlts}

A historical record of real a transformer is included here for proof-of-concept. Fig. 6 shows a transformer that has been anonymised but still has its indicative gas trends left intact. This can be used as an approximate point of reference to correlate the outputs against.

As mentioned previously, Fig. 5 is an output of the diagnostic analyses when a sample is classed as a Status Level 2 or 3 . It shows that initially a mixture of electrical and thermal faults was suspected until around sample 1000, where a thermal fault was also suspected. During this time, Duval Triangle 4 also indicated potential stray gassing of oil. The Rogers Ratio method does not have complete 'coverage' of all gas ratios, and can often result in no outputs [1], which was the case for this case study.

Fig. 7 shows the Status Level for each sample. It is argued that too much information is lost when solely using the Status Level. This is evidenced by the Status Level remaining constant for almost the entire duration of the case study. The next subplot shows three possible implementations of the derived Health Index. This includes using the worst-case Status Levels, the average Status Levels, and the proposed average of both the worst-case and average Status Levels. It is recommended that once the Health Index suggests an issue has arisen, to then delve into the individual gas outputs for further information. 


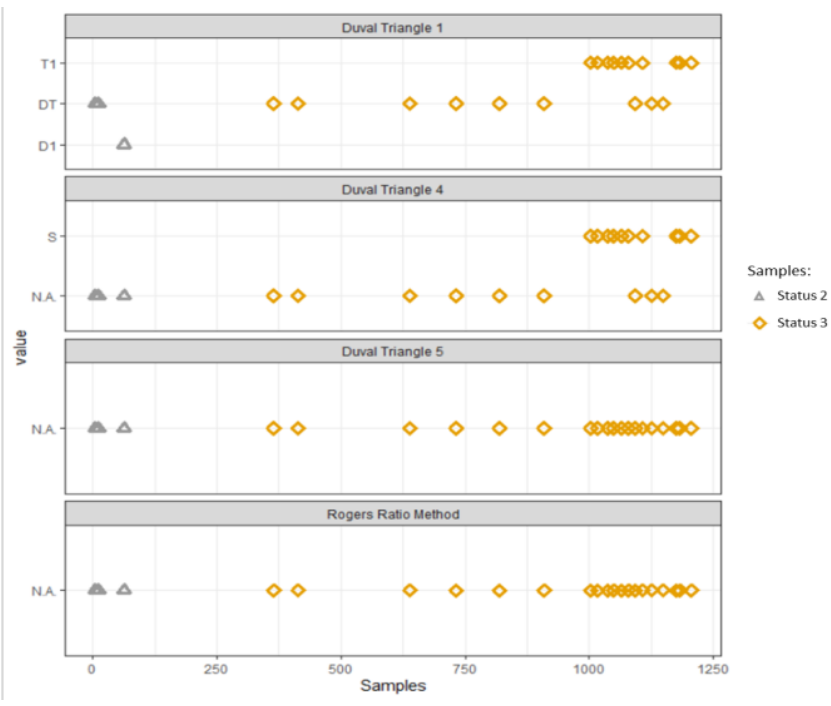

Fig. 5. Case study's Duval Triangles 1-4-5 and Rogers Ratio outputs

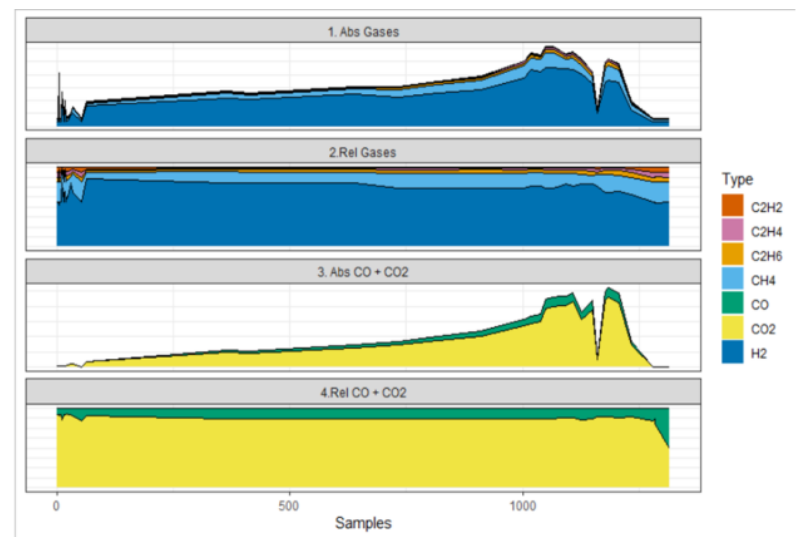

Fig. 6. Case study's anonimised gas levels

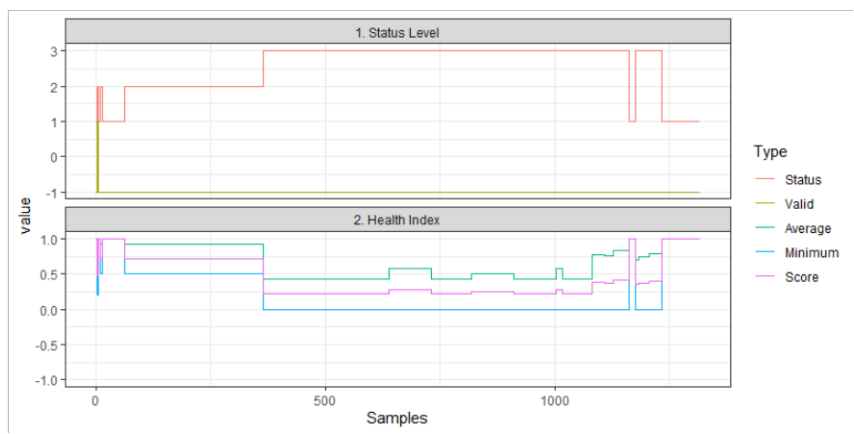

Fig. 7. Case study's Status Level and corresponding Health Index

\section{DISCUSSION}

The IEEE Standard C57.104-2019 provides invaluable insight into a substantial dataset of transformers via the percentile-based thresholds presented in the Guide's Tables. It would have been even more useful were the anonymised distributions of said metrics, or even the raw gas levels provided instead. Having access to these distributions would have allowed for the fine-tuning of the percentile-based thresholds to better accommodate a given risk appetite.

The thresholds suggested for acetylene for Table 3 and Table 4 are prone to erroneous flagging due to measurement noise. These Tables relate to the relative change in gas levels between subsequent samples, and a projected annualised changed in gas levels based on a specific selection of previous samples, respectively. Both are set to 0 , i.e. any increase is flagged at a Status Level 3. It is challenging to address this as expected acetylene levels are often very low and increases to the threshold may cause undue desensitisation to potentially significant increases.

The sampling criteria for Table 4 seem too restrictive for the method developed in this paper. It is not clear why an upper bound to the number of samples within a given period is given. With this system, erratic sampling frequency rates can lead to Table 4 becoming inapplicable at times which can affect the Status Level.

Guidance related to the Duval Triangle and Rogers Ratio method suggest using the relative changes in gas levels over a given timespan to improve sensitivity to recent developments within the Transformer [1], [2], [5], [6]. However, this can be challenging to implement in an automated manner as the relevant timespan varies contextually.

\section{CONCLUSIONS}

An automated implementation of the IEEE Standard C57.104-2019 has been developed. This includes two diagnostic analysis methods: the Duval Triangles 1-4-5 and Rogers Ratio method. Furthermore, a proposed Health Index derived from the Status Level outputs of the Guide was implemented and demonstrated. It was shown that generally the developed derived Health Index with corresponding Transformer diagnosis seems to maintain a reasonable balance between being informative, compact, and traceable back to the original metrics. Future work will involve incorporating additional analyses techniques within this framework to provide a more comprehensive Health Index.

\section{ACKNOWLEDGMENT}

This work was funded by the University of Strathclyde's Advanced Nuclear Research Centre in collaboration with EDF Energy and Bruce Power.

\section{REFERENCES}

[1] IEEE Std C57.104 $4^{\mathrm{TM}}-2019$, IEEE Guide for the Interpretation of Gases Generated in Mineral Oil-Immersed Transformers, vol. 1991. 2019.

[2] IEC 60599, "Mineral oil-impregnated electrical equipment in service - Guide to the interpretation of dissolved and free gases analysis," Iec 60599, vol. 3, pp. 1-69, 2015.

[3] T. K. Saha, "Review of Modern Diagnostic Techniques for Assessing Insulation Condition in Aged Transformers," in IEEE Transactions on Dielectrics and Electrical Insulation, 2003, vol. 10, no. 5, pp. 903917, doi: 10.1109/TDEI.2003.1237337.

[4] R. R. Rogers, "Ieee And IEc Codes To Interpret Incipient Faults In Transformers/ Using Gas In Oil Analysis," IEEE Trans. Electr. Insul., vol. EI-13, no. 5, pp. 349-354, 1978, doi: 10.1109/TEI.1978.298141.

[5] M. Duval, "A review of faults detectable by gas-in-oil analysis in transformers," IEEE Electrical Insulation Magazine, vol. 18, no. 3. pp. 8-17, 2002, doi: 10.1109/MEI.2002.1014963.

[6] M. Duval, "The duval triangle for load tap changers, non-mineral oils and low temperature faults in transformers," IEEE Electr. Insul. Mag., vol. 24, no. 6, pp. 22-29, 2008, doi: 10.1109/MEI.2008.4665347. 